Paulette ROULON-DOKO*

\title{
La chèvre dans une société de chasseurs- cueilleurs-cultivateurs l'exemple des Gbaya 'bodoe de R.C.A.
}

\begin{abstract}
Résumé
L'auteur présente le statut de la chèvre ou cabri chez les Gbaya 'bodoe, une société de chasseurs-cueilleurs-cultivateurs vivant en République Centrafricaine. Après avoir mentionné les dénominations et les termes qui s'appliquent aux cabris, elle décrit la façon dont les Gbaya s'en occupent avant de préciser le rôle qu'ils jouent dans leur société, soulignant leur importance dans la constitution de la compensation matrimoniale. Dans l'univers des contes, c'est d'ailleurs comme élément fondamental de la compensation matrimoniale que les cabris interviennent. Leur vie confinée au village en fait le symbole de l'endogamie et, dans l'imaginaire, ils sont une nourriture convoitée.
\end{abstract}

Mots-clefs

R.C.A., Gbaya, cabri, compensation matrimoniale.

\footnotetext{
* Directeur de Recherche au CNRS, LLACAN-INALCO, roulon@vjf.cnrs.fr
} 
Dans une société de chasseurs-cueilleurs-cultivateurs comme celle des Gbaya $^{1}$ de la République Centrafricaine, l'élevage joue un rôle très marginal et ne concerne que trois animaux, le chien, la poule et la chèvre. De fait ces trois animaux n'ont pas le même accès à l'espace : la poule ne quitte jamais le village, tandis que le cabri se promène volontiers dans la savane jouxtant le village et que le chien parcourt toute la brousse à l'occasion des chasses ${ }^{2}$. Ils occupent, d'ailleurs, chacun une position bien différente dans la société. Le chien est l'auxiliaire privilégié du chasseur, la poule a surtout un rôle rituel $^{3}$ et la chèvre est un élément important de la compensation matrimoniale. C'est à la chèvre, également appelée cabri, que cet article est consacré.

\section{LES DENOMINATIONS DU CABRI}

Le terme dù $\grave{a}^{4}$ « chèvre, cabri » est commun à toute l'aire gbaya. Il a été reconstruit en proto-gbaya par Yves Monino (1995: 609). Philip Noss, pour le gbaya yaayuwee mentionne un synonyme mbúdì qu'il signale comme un emprunt au bantou. Ce même terme est cité par Yves Monino pour un certain nombre de dialectes de la zone sud de l'aire Gbaya-ManzaNgbaka. En gbaya 'bodoe par contre, le terme dùà a comme synonyme bòtádò qui n'a été signalé dans aucun des autres parlers.

Les Gbaya considèrent qu'il n'existe qu'une seule «race, lignage » zúdùk (sommet/à rester) de cabri. Cependant, un vocabulaire spécifique

\footnotetext{
${ }^{1}$ Les populations qui se reconnaissent sous le nom de Gbaya occupent un territoire situé pour les quatre cinquième à l'ouest de la République Centrafricaine et pour le dernier cinquième au centre-est du Cameroun. L'étude présente porte plus précisément sur les Gbaya 'bodoe qui font partie du groupe numériquement le plus important (160000 locuteurs) les Gbaya kara. Ils forment un groupe homogène d'environ 5000 personnes réparties en une quarantaine de villages au sud-ouest de Bouar, en République Centrafricaine. La langue gbaya appartient, selon la classification de Greenberg, au groupe 1 de la branche orientale de la sous-famille 6 "Adamawa oriental" de la famille Niger-Congo, plus volontiers appelé Oubanguien dans les études plus récentes.

${ }^{2}$ Ce qu'illustre bien le conte de la jeune fille Sárá-kò-bérà dont il faut découvrir le nom pour pouvoir l'épouser. Un coq, un chien et un cabri viennent pour l'épouser et ce sera le coq qui, lui seul, saura découvrir le nom, soulignant en cela que si la poule a un univers spatial plus réduit, elle en a par contre une meilleure connaissance.

C'est l'aliment rituel par excellence, essentiellement dans l'établissement des relations d'alliance et la prière du chef de famille aux ancêtres. Elle sert également de substitut à l'individu lors des procédures ordaliques. $C f$. Roulon-Doko, 2008 : 560-561.

${ }^{4}$ Un terme homonyme dùà désigne « la sorcellerie ». Blanchard et Noss (1982 : ) font, pour le Gbaya yaayuwee, un rapprochement entre les deux et parlent du cabri comme d'un animal de sorcier. En gbaya 'bodoe, le terme « sorcellerie » a deux réalisations attestées comme des variantes libres d’̀a ou dùa . Le rapprochement formel entre la deuxième réalisation et le terme «cabri » n'est jamais effectué par les locuteurs et je n'ai relevé aucun discours qui rapprocherait le cabri du sorcier.
} 
prend en charge les différences de taille et de couleurs comme leurs traits les plus caractéristiques. Ainsi un cabri qui est «moitié blanc, moitié noir » est appelé bìr-kòtó ${ }^{5}$ (à couper / peau). Il n'est pas rare qu'un cabri ait une " tâche blanche » fóré sur la face. Un bouc qui ne grandit pas et reste de petite taille est appelé « un petit bouc » ngbókó, tandis qu'on parle d' « un bouc bien outillé » ngàk-ngék vàlá dùà (pour impressionner / mâle.D /cabri) quand il a de fortes cornes. Un petit chevreau saute "pattes jointes» kàràk kàràk ${ }^{6}$ comme un céphalophe bleu et une grosse brebis marche "lourdement» $y \grave{\varepsilon} r \grave{\varepsilon} r \grave{\varepsilon}$. Les cabris sont réputés pour avoir un ventre «bien gonflé » búytúy et on dit aussi qu'ils « mastiquent très longuement » leur nourriture dùà jóy gèdà mùr mùr mùr (cabri/ INAC.manger/ manioc/ en mastiquant). D'ailleurs, le conte de "La cola de la panthère » górò kó gd montre comment un cabri est accusé du vol des cola, car sa façon de mastiquer sans arrêt l'a désigné comme tel. Un autre élément caractérise les cabris, c'est la forme de leurs «excréments » ďr $r$ dùà qu'on rendra plus spécifiquement par «crottes de bique». C'est ainsi que deux plantes sont nommées par référence aux crottes de bique. Le Leea guineensis LEEACEAE dont «le fruit, rouge brillant puis noir est globuleux et bosselé de $1 \mathrm{~cm}$ de diamètre» (Letouzet, 1972 : 250,254) est appelé en gbaya gbàràà-dùà (mettre bout à bout / cabri), car ses fruits sont collés les uns aux autres comme les crottes de cabri ; et une herbe de savane (CYPERACEAE ?) qui reçoit le surnom de « crottes de cabri » ď̌r-dùà (excrément.D / cabri) car sa racine y ressemble.

En gbaya 'bodoe, au sein des cabris, on distingue, selon le genre, entre les femelles appelées «chèvres" nàà kó dùà (mère/de/cabri) et «chevrettes » zóná dùà (jeune fille. $\mathrm{D}^{7}$ / cabri) pour les toutes jeunes et les mâles appelés «boucs » bàfá / vàlá dùà (mâle.D / cabri). Tandis que le terme bàfà désigne génériquement le mâle de n'importe quel animal, le terme vàlà ne s'applique qu'aux caprins et aux ovins ${ }^{8}$. Les " chevreaux », mâles ou femelles, sont appelés bé dùà (petit.D / cabri) pour les tout petits et bé-dáyá dùà (petit-haut.D/cabri) quand ils sont un peu plus grands. Lorsqu'une femelle ne met jamais bas, on la dit «stérile » bònd’̀r̀̀. Il arrive aussi qu' « on castre un bouc » Péí kàyà fálá dùà (on / ACC.ramasser.D / testicule.D / cabri) pour qu'il grossisse bien.

Contrairement aux chiens, les cabris ne reçoivent pas de noms propres. Cependant le comportement caractéristique de certains cabris reçoit une

\footnotetext{
${ }_{6}^{5}$ Ce même terme peut caractériser un chien ou une vache.

${ }^{6}$ Cet adjectif-adverbe kàràk qui signifie « formant un bloc », caractérise aussi les sauts du céphalophe bleu.

${ }_{8}^{7}$ Signale la présence d'un ton haut représentant le morphotonème relationnel.

${ }^{8}$ Le mouton que les Gbaya connaissent pour l'avoir vu chez des les Peuls mbororo est désigné par le terme sámbí. Il n'y a pas d'élevage de moutons chez les Gbaya.
} 
dénomination spécifique ${ }^{9}$ qui peut désigner à l'occasion un cabri qui se comporte ainsi. Ainsi on appellera «monte aux poteries» dàn-kpánà (à monter / poterie) le cabri qui fait des dégâts dans la maison, car il cherche toujours à voir ce qu'il y a au fond des diverses poteries ${ }^{10}$ qui sont rangées dans la maison. L'appellation de "fonce dans la farine» yùr fùù (à foncer / farine) désigne un cabri qui se précipite si vite lorsqu'il sent du manioc qu'il risque de tout culbuter sur son passage, et celle de " casanier » kpélém un cabri qui reste toujours au village, à l'affût d'une porte ouverte. Enfin on appellera la «mère de la bergerie » nàná-gbày (mère.D / bergerie) une femelle très prolifique, qui met toujours au monde des agneaux, deux par deux. On emploie dans ce cas non plus le verbe «mettre au monde » ko, mais le verbe « verser » gbai, qui s'utilise également pour une femme qui a mis au monde des jumeaux.

\section{L'ENTRETIEN DES CABRIS}

Les cabris font partie des animaux que les Gbaya élèvent et dont ils s'occupent depuis très longtemps. Autrefois, lorsqu'il n'était pas rare qu'une panthère rôde autour du village, chaque lignage construisait alors d'une « bergerie » gbăy dùà (enclos.D / cabri), dans laquelle étaient rentrés tous les soirs les cabris. Il s'agissait d'une véritable maison, construite en terre et couverte d'un toit de chaume. La porte se composait d'une série de troncs de bois entassés les uns sur les autres et maintenus par deux gros poteaux. Chaque matin, pour l'ouvrir, on ôtait les bois du dessus, en en laissant toujours deux en bas. Les cabris sortaient en sautant par dessus. Dans la bergerie, plusieurs piquets coudés étaient fichés au sol. Cela permettait d'attacher, par une corde ficelée à une de leurs pattes avant, les femelles les plus belliqueuses ou parfois un mâle trop querelleur. Lorsqu'une panthère s'était approchée du village au point d'avoir pu blesser un cabri, les villageois pour mettre un terme à la menace que représentait ce fauve fabriquaient un «piège à panthère » śé $\eta \dot{\varepsilon} n$ (sagaie/ en haut) littéralement 'les sagaies en l'air'. Pour ce faire, ils récupéraient le cabri blessé et le suspendaient à une branche haute d'un arbre, en bordure du village. En dessous, ils fichaient des sagaies, pointe en l'air, de façon à faire un tapis continu de pointes qui, empêchant la panthère d'atteindre sa proie, l'incitait à sauter pour parvenir à son but. En retombant, elle s'embrochait sur les sagaies. L'extermination quasi complète des panthères par les fusils

\footnotetext{
${ }^{9}$ Il s'agit d'une dénomination de type.

${ }^{10}$ Il s'agit en particulier de la très grande poterie dans laquelle est conservée le manioc en cossette et la poterie de taille moyenne où l'on conserve la farine de manioc des prochains repas. Pour parvenir à ses fins, un tel cabri renverse ces poteries qui sont simplement posées sur un trépied.
} 
des Blancs installés à Bouar, au début du $\mathrm{XX}^{\mathrm{e}}$ siècle, a rendu caduque cet abri et ce type de piège. La bergerie ne servait de protection contre la panthère que pendant la nuit. De jour, les cabris étaient lâchés et se déplaçaient librement dans la savane avoisinant le village à la recherche de l'herbe pour se nourrir, comme ils le font toujours de nos jours. Les cabris n'ont jamais fait l'objet en pays gbaya d'un quelconque affourragement en stabulation et n'ont jamais été accompagnés dans leurs déplacements.

De nos jours, les cabris se nourrissent au village et aux abords du village, circulant aussi dans la brousse, jusqu'aux rivières et aux aires à manioc ${ }^{11}$ les plus proches. Ils ne s'aventurent jamais très loin en brousse et ne vont, en particulier, jamais jusqu'à l'aire des champs, toujours située assez loin du village ${ }^{12}$. De fait, chaque année, de nouveaux champs destinés au sésame sont débroussés les uns à côté des autres formant une aire bien circonscrite bien qu'aucune clôture n'en marque les limites. Au village même ils sont à la recherche de certains résidus alimentaires qu'on jette et mangent l'herbe des portions qui séparent les quartiers dont le sol est par contre entièrement désherbé et nu. Si quelqu'un fait un jardin auprès de sa maison, il ne manque pas de le clôturer pour empêcher les cabris d'aller s'y repaitre. Ils dorment à la belle étoile, le plus souvent collés au mur d'une maison surtout lorsqu'il pleut.

\section{L’UTILISATION DES CABRIS}

Il n'y a chez les Gbaya 'bodoe aucune utilisation du lait que produisent les chèvres. Il est entièrement laissé aux chevreaux. Seules la viande et la peau du cabri sont utilisées.

Dans l'alimentation quotidienne, on mange peu de cabri et on dit que

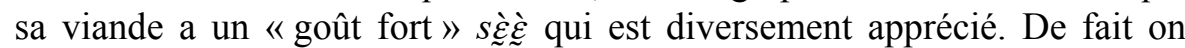
ajoute volontiers à la cuisson de la viande de cabri un bouquet de Cymbopogon giganteus fólópèy qui en atténuera le goût. Certaines femmes en particulier trouvent le goût du cabri si prononcé qu'elles n'en mangent jamais, elles préfèrent de loin la viande des animaux sauvages (rongeurs, céphalophes, suidés, guibs, etc.). Comme dans le cas des gibiers ${ }^{13}$, les Gbaya consomment les tripes du cabri. Mais, ce n'est que dans le cas du cabri, que les boyaux sont tressés ( $c f$. Roulon-Doko, $2001: 148$ ) ce qui les rend, dit-on, plus facile à saisir lorsqu'on les mange. C'est donc quand il

\footnotetext{
${ }^{11}$ Ce sont des pierres de granit naturel qui servent à faire sécher le manioc qui a été roui. Il y reste toujours des résidus de manioc dont les cabris sont très friands.

${ }^{12}$ Les champs sont éloignés d'une distance comprise entre 3 et $5 \mathrm{~km}$, soit une marche de $1 / 2$ heure à 1 heure.

${ }^{13}$ A l'exception des tout petits rats et des oiseaux dont on jette les intestins.
} 
convient de nourrir, de façon impromptue, un groupe important de visiteurs qu'on tuera un cabri. C'est le cas par exemple au moment d'un deuil, lorsque les parents se rassemblent dès qu'ils ont appris la nouvelle de la mort. Par contre, lorsqu'une fête est prévue à l'avance, on préférera préparer du gibier, frais ou boucané, et de nos jours, on achètera de la viande de vache. Lorsque quelqu'un rassemble des gens pour labourer son champ, il se doit d'offrir un repas à la fin de la journée. Un cabri peut alors être tué et préparé pour cela.

Tout cabri tué pour être consommé est systématiquement "dépecé » báá kòtó dùà yúné (dépecer / peau.D / cabri / en ôtant). La peau est ensuite râclée et mise à sécher au soleil. C'est toujours une peau de cabri qui recouvre les deux faces du «tambour à deux peaux » dàyírì. Par contre pour le «tambour à pied " gàtà, c'est soit une peau de cabri soit une peau de céphalophe roux qui est utilisée. La peau de cabri sert également pour faire l'assise des petits fauteuils en bois que certains fabriquent.

\section{LA CONSOMMATION RITUELLE DU CABRI}

Je vais présenter les deux circonstances précises où un cabri est rituellement tué chez les Gbaya.

Chaque année, au début de la saison sèche, avant la première chasse au feu, on adressait une prière aux ancêtres en leur demandant protection et bienveillance pour tous. Cette prière était effectuée par le maître de la terre pour l'ensemble des territoires de chasse du village. Celui-ci appartenait toujours au lignage dont le territoire en question était le territoire ancestral. C'était la seule prière aux ancêtres qui concernait l'ensemble des villageois, les autres étant toujours des prières plus restrictivement adressées aux ancêtres d'un seul lignage. Un cabri était alors sacrifié. Il était, comme tous les autres cabris qu'on mange, égorgé avant d'être dépecé. Il représentait la fécondité $^{14}$ du village et était consommé par les neveux utérins et les gendres, tandis que les autres villageois consommaient, eux, des termites ou des champignons séchés avec de la pâte de sésame. Un morceau du cabri cuit, de la pâte de sésame et un peu de boule de manioc était ensuite laissé dans un plat au pied de l'arbre où avait été fait la prière, à l'intention des ancêtres. C'est par contre un poulet qui est sacrifié pour toutes les autres offrandes individuelles faites aux ancêtres. Il semble donc que c'est parce

\footnotetext{
${ }^{14}$ Le principe de cette consommation rituelle par un individu ou un groupe précis est de réactiver la fécondité spécifique qui est ensuite rendue à l'intéressé par l'imposition d'une marque. Pour une présentation détaillée des rites liés à la fécondité chez les Gbaya, $c f$. Roulon-Doko, 1998 : 203-214 et $2001: 298-301$.
} 
qu'il faut une grande quantité de viande pour permettre la consommation de tous les utérins et alliés qu'on tue un cabri et non un poulet.

D'une manière générale, c'est le sacrifice d'une poule qui marque les différentes étapes du mariage ${ }^{15}$ (cf. Roulon-Doko, 2001 : 299-300). Ainsi, de façon régulière c'est à la poule dite «de séparation"kìrá-kàfì-tè, offerte aux beaux-parents le jour de la conduite effective de l'épouse chez son mari, que revient le soin de marquer qu'il y a bien eu séparation de l'épouse d'avec le lignage de son père. Cependant lorsqu'une jeune femme a fait plusieurs fausses couches ou ne parvient pas, plusieurs fois de suite, à mettre au monde un enfant viable, les Gbaya considèrent ce problème comme le signe d'une séparation qui n'a en fin de compte pas été bien marquée. Pour y remédier, le mari tuera pour sa belle famille un cabri. Pour ce rituel dit « du cabri sacrifié » dùá-gb̀̀, le cabri n'est pas comme égorgé, comme on l'a vu jusqu'ici, mais tué en lui tordant le cou. Ce cabri cuit avec un peu de pâte de sésame et un bouquet de Cybopogon giganteus est consommé par les tantes paternelles et les pères classificatoires de l'épouse. L'un d'entre eux tracera avec un peu de sang dudit cabri un trait sur le corps de la jeune femme afin qu'elle retrouve sa fécondité et puisse ensuite mettre au monde un enfant viable. Le cabri prend ici le relais de la poule, pour donner un signe fort de la séparation nécessaire entre l'épouse et sa famille, puisque celle-ci n'a pas eu les effets attendus. Je pense que le cabri auquel on a recours permet de partager ce repas entre plus de parents maternels que la poule dont la consommation est, chez les Gbaya réservée aux seuls hommes. En agrandissant le cercle des parents impliqués dans ce rituel on augmente les chances d'en avoir satisfait le plus grand nombre et donc d'apaiser les ancêtres qui, seuls, peuvent disposer de la fécondité ${ }^{16}$ de leurs descendants et donc sont nécessairement à l'origine du dysfonctionnement constaté.

\section{LES CABRIS DE LA COMPENSATION MATRIMONIALE}

Mais l'intérêt que portent les Gbaya aux cabris est surtout lié au rôle qu'ils jouent dans la «compensation matrimoniale» mbj̀í-dòy-kóò (monnaie.D / derrière.D / épouse) que le futur gendre doit verser à sa belle famille pour valider le mariage. La compensation matrimoniale se compose d'un certain nombre de cabris - de dix à trente selon les cas - et, autrefois,

\footnotetext{
15 Il n'y a aucun conjoint prescrit ou préféré. Sont interdits tous les parents du lignage du père et de la mère ainsi que ceux issus du lignage des quatre grands-parents (PP, MP, PM et $\mathrm{MM}$ ).

${ }^{16}$ La fécondité et en particulier celle des femmes liée à la progéniture est expressément placée sous le contrôle des ancêtres auxquels on pense en premier dès que quelque chose ne semble pas tourner rond.
} 
de barres de fer ${ }^{17}$ qui sont aujourd'hui remplacées par une somme d'argent ${ }^{18}$.

Dans un conte, le beau-père qui, souffrant de rhumatismes, ne peut plus marcher, confie à l'écureuil que s'il lui trouve une monture pour se déplacer, il le dispense de toute compensation matrimoniale et s'engage à lui donner sa fille. Il précise bien : "Tu n'auras pas à verser d'argent, ni à donner de cabris » $m \varepsilon$ há mbòi ná Tòé, mé há tí dùà ná ná iòé, soulignant bien la complémentarité de ces deux éléments - «argent» mbj̀ et « cabris » dùà - dans la constitution de la compensation matrimoniale traditionnelle. C'est ainsi qu'à chaque mariage, quelqu'un «compte les cabris de la compensation matrimoniale » Pà tòrá dùà dòy kóò (il/ ACC.compter/ cabri / derrière.D / épouse). A titre d'exemple, je mentionnerai que la compensation matrimoniale versée pour l'épouse lors d'un mariage, au village de Ndongué, le 6 mai 1970, se composait de 10 cabris, $8500 \mathrm{~F}$. CFA, 1 pagne, 1 petit boubou et 250 F. CFA pour le jour même de la conduite de l'épouse au village du mari.

Les éléments qui composent la compensation matrimoniale ne produisent pas, chez les Gbaya, une situation qui puisse rendre dépendant le jeune homme qui veut se marier. De fait les cabris font partie des biens que tout jeune garçon acquiert petit à petit depuis sa naissance. Il reçoit ainsi à différentes occasions informelles des cabris qui lui sont offerts par les frères de son père et également par ses oncles maternels. C'est le plus souvent une jeune chèvre qui est offerte. Elle va bien sûr mettre bas et contribuer par ses petits à accroître le troupeau. Tous ces cabris viennent grossir le troupeau de son père qui, le jour de son mariage, lui rendra ses cabris et y ajoutera quelques uns des siens. Chaque père de famille dispose donc d'un troupeau et le troupeau collectif d'un segment de lignage - de 10 à 30 personnes ${ }^{19}$ peut atteindre une centaine de cabris. Chacun sait très bien reconnaître ses propres cabris et en fait ce qu'il veut. Les cabris peuvent être donnés ou vendus, en fonction des besoins de chacun. Les cabris sont donc un élément essentiel de la compensation matrimoniale et sont également un cadeau que peut offrir un gendre à ses beaux-parents.

\footnotetext{
17 C'était le lignage du père qui fournissait les barres de fer produite au village par l'extraction du minerai. Cette extraction ( $c f$. Monino, XX) qui n'était pas le fait de spécialistes était cependant placée sous la responsabilité des ancêtres. Elle permettait un approvisionnement régulier en fer et les barres de fer constituaient une richesse collective qui circulait régulièrement entre les lignages à l'occasion des mariages.

18 Depuis l'introduction de l'argent, les jeunes gens ont pris l'habitude d'aller s'employer en ville afin de se constituer le pécule qui leur permettra de verser l'argent de la compensation matrimoniale. Leurs parents peuvent les aider, mais le plus souvent on attend d'eux qu'ils cherchent eux-mêmes l'argent dont ils auront besoin.

${ }^{19}$ Ce qui représente de 1 à 5 ou 6 chefs de famille.
} 


\section{DE L'ORIGINE DES CABRIS}

Il existe un conte $^{20}$ qui relate l'origine de la compensation matrimoniale réduite ici à la constitution d'un troupeau de cabris dont voici le résumé :

Wanto, qui posait en vain des pièges en brousse sans parvenir à constituer la compensation matrimoniale qu'il lui fallait pour se marier, eut recours à la ruse. Il captura une jeune femelle de céphalophe couronné qu'il fit passer pour une 'chèvre' auprès de Gbason, lui demandant de la garder avec les siennes jusqu'à ce qu'elle fasse ses petits. Six mois plus tard, lorsque Wanto vint reprendre sa 'chèvre' et les petits qu'elle n'avait pas manqué d'avoir, Gbason lui assura que sa chèvre s'était enfuie le soir même où il l'avait mise dans sa bergerie. Ne pouvant se mettre d'accord, ils demandèrent au chef du village de les départager. Au cours du jugement, Wanto précisa bien que sa 'chèvre' avait une marque noire sur l'oeil. Comme Gbason ne put pas expliquer pourquoi il n'avait pas prévenu Wanto de la disparition de sa chèvre, le juge donna raison à Wanto. Pour le dédommager il lui dit d'emporter tous les cabris qui avaient une marque noire à l'oeil. C'est ainsi que Wanto constitua ainsi la première compensation matrimoniale.

Il n'est pas question dans ce conte de la domestication d'une espèce sauvage ${ }^{21}$ comme dans les récits de montagnards du Cameroun, mais bien d'une ruse qui, induisant en erreur le seul propriétaire de cabris Gbason, l'oblige à en donner à Wanto qui, comme tous les autres hommes, n'en avait encore jamais possédé. Le céphalophe couronné, Sylvicapra grimmia, a de fait une allure générale assez différente des autres céphalophes. Il est d'une couleur très variable, avec un dos presque droit, des pattes plus longues et des cornes verticales ouvertes en $\mathrm{V}^{22}$. L'ensemble lui donne une allure qui peut rappeler celle du cabri, ce qui le rend particulièrement apte à produire la méprise voulue par Wanto. On voit dans ce récit l'importance accordée au signe particulier mentionné par Wanto (une marque noir sur l'œil) qui devient un signe identificateur faisant reconnaître, sans hésitation, tous les cabris qui ont cette marque comme les petits de sa prétendue chèvre. Gbason est, dans les contes gbaya, le possesseur de tous les biens (manioc, sésame, eau, cabris) dont les hommes étaient en ces temps-là privés. C'est toujours par la ruse que Wanto, le héros civilisateur des contes gbaya, parvient à acquérir ces biens pour en faire profiter tous les hommes. L'acquisition du cabri ne relève donc pas d'un procédé de domestication, mais du vol d'un animal déjà domestiqué dans la situation initiale, au seul profit de Gbason. Le cabri est donc ici perçu comme un bien, avant d'être un animal.

\footnotetext{
${ }^{20}$ Une version de ce conte a été publiée dans Roulon, 1977 : 28-33.

${ }^{21}$ Gazella rufifrons selon les informations recueillies par Christan Seignobos et mentionnées dans ce dossier.

${ }^{22}$ Description reprise d'après Dorst et Dandelot, $1972: 260$ ssq.
} 


\section{DIRES ET CONCEPTIONS SUR LES CABRIS}

Le confinement des cabris au village et à ses proches alentours en fait un symbole d'endogamie. Lors d'un jugement coutumier qui confrontait un jeune couple avec la famille de l'épouse qui leur demandait des comptes, du fait qu'ils avaient déjà, sans être officiellement mariés ${ }^{23}$, deux enfants, la situation était ainsi exprimée: "Vous agissez l'un et l'autre sans discernement, comme un bouc et une chevrette ». En effet, l'attitude des cabris qui vivent au village et se reproduisent entre eux symbolise aux yeux des gbaya une attitude a-sociale qui n'est pas acceptable. Le conte du « travail du gendre du bouc » néć kj̀fê kó vàlá dùà (aller / travail du gendre / $\mathrm{du} /$ mâle.D / cabri) propose une explication pour ce comportement du cabri.

Un bouc part chez ses beaux-parents pour y faire le travail du gendre. Sur son chemin il rencontre une panthère qui l'informe qu'elle l'attendra à son retour. Mort de peur, il écourte sa visite et revient au plus vite pour ne plus jamais quitter son village où depuis il prend femme entre ses soeurs et sa mère.

En dehors de son rôle comme élément fondamental de la compensation matrimoniale, le cabri est mis en scène dans deux contes où il représente un bien désirable. Dans le conte sur "l'origine de la mort», Wanto vient emprunter à la Mort une chèvre qu'il promet de rendre une fois qu'elle aura mis bas. La Mort est présentée comme quelqu'un de riche, tandis que Wanto n'a pas grand chose et il est en l'occurrence bien précisé que ce qui manque à ce dernier ce sont des cabris.

Tout ce qu'on peut trouver, la mort le possède, Wanto n'est pas quelqu'un qui trouve grand chose. Ce qui manque à Wanto ce sont des cabris.

La notion de « richesse » kpáá mò (nv.trouver / chose), littéralement 'le fait de trouver quelque chose' prend en compte tout ce qu'une personne peut trouver. Cela comprend en tout premier lieu pour un homme, les gibiers qu'il peut tuer à la chasse ou par piégeage. Or, pour les Gbaya, le gibier est directement placé sous le contrôle des ancêtres avec lesquels il faut être en bons termes pour qu'ils vous aident dans toutes ces activités, ce que manifestent bien tous les rites de fécondité à leur propos ${ }^{24}$. Les autres produits de cueillette, comme les produits cultivés et les animaux qu'on élève, participent bien sûr à cette richesse mais ne dépendent pas des ancêtres, aucun rite ne s'y rattachant. La richesse est donc perçue par les Gbaya comme l'aptitude à pouvoir disposer régulièrement de ce dont on a besoin pour vivre en le partageant au sein de sa famille et pas comme la

\footnotetext{
${ }^{23}$ C'est à dire qu'aucune compensation matrimoniale n'avait été versée.

24 Les femmes ont également des gibiers qu'elles tuent pendant une chasse qui leur est spécifique et pour lesquels il y a également des rites de fécondité, $c f$. Roulon-Doko, Réf.
} 
possibilité d'une accumulation. Une telle accumulation individuelle place celui qui la réalise comme une personne hors normes sociales ce que Gbason, unique possesseur de cabris et d'autres choses, illustre bien. D'une manière générale toute thésaurisation d'un bien quelconque met en cause la régulière circulation et le partage que le groupe est en droit d'attendre de chacun des siens ${ }^{25}$ et est perçu négativement par les Gbaya.

La relation de prêt s'établit au cours du dialogue suivant entre Wanto et la Mort.

- La Mort, je n'ai pas de cabris! Petit-père donnez ${ }^{26}$ m'en donc un que je m'en occupe moi aussi, parce que vous, vous avez ici des choses à élever, moi je n'en ai pas du tout.

Mais la Mort rétorque :

- Est-ce que tu as de quoi payer?

- Non, je n'ai pas d'argent, je te demande de me le donner gratuitement seulement.

- Est ce que tu viens demander un emprunt ?

- Oui, je te le demande comme un emprunt.

La mort prend un cabri et le lui donne [demandant:]

- Quand le lui rendra-t-il ${ }^{27}$ ?

- Quand ledit cabri aura mis bas, il lui rendra seulement le petit en retour.

La mort dit que puisqu'ils vivent ensemble au village, ils doivent en fin de compte s'entraider. Mais pour le lui rendre, il ne veut pas que ça dure longtemps. Aussi une fois que ledit cabri aura mis bas, qu'il lui rende un des cabris et chacun en aura ainsi un pour soi. Wanto est d'accord et prend sur le champ le cabri.

Le fait de prêter ou de confier un animal pourrait être comparée à la situation de la vache d'attache dont nous parle Jean Boutrais qui précise que «ces animaux à présence temporaire participent à une véritable circulation du bétail au sein de la société pastorale». Mais ici, l'éventualité d'une telle circulation est aussitôt annihilée par le comportement de Wanto qui s'empresse de tuer la chèvre prêtée pour la manger avec son épouse. Dans la vie quotidienne, je n'ai jamais entendu parler de telles pratiques.

Enfin, dans un autre conte, Bidiwiri-bidikpa se promène avec un bouc qu'il propose à tous ceux qu'il rencontre comme récompense contre une victoire à la lutte. Son cri d'appel est « un cabri contre un combat !». Wanto accepte aussitôt le pari et consomme l'animal dès qu'il lui est donné, avant

\footnotetext{
25 Dans un conte, Wanto ruse pour être le seul consommateur de tous les gibiers qu'il prend dans ses pièges en en privant ainsi sa femme et son fils. Il est, dans la suite du conte, fortement condamné pour un tel comportement. ( $C f$. Roulon-Doko : ).

${ }^{26}$ Le vouvoiement qu'emploie Wanto pour s'adresser à la Mort est de règle entre les parents lorsque quelqu'un s'adresse à quelqu'un d'une génération supérieure à la sienne ou à un ou une aîné(e) dans sa propre génération. Wanto se place vis-à-vis de la Mort comme un fils vis-à-vis de son père. Celui-ci lui répond en employant le tutoiement. Chez les Gbaya, en dehors des membre de la famille, on a recours à un tutoiement réciproque.

${ }^{27}$ Le passage à la troisième personne cherche à rendre l'usage du logophorique employé ici en gbaya au lieu des pronoms employés jusque là.
} 
même d'avoir combattu. Battu ensuite à la lutte, il ne peut, comme dans le cas de la chèvre prêtée par la Mort, rendre l'animal.

La mention du cabri, dans ces deux contes, le montre comme un objet convoité dans le seul but de satisfaire un appétit glouton plutôt jugé négativement, puisque dans les deux cas, Wanto se retrouve dans une position dramatique dont l'issue est la mort à laquelle il succombe dans le premier conte et à laquelle il échappe de justesse dans le second.

\section{EN CONCLUSION}

L'entretien des cabris ne représente pas une grosse charge de travail et le développement du troupeau familial se fait petit à petit, permettant à chaque garçon en âge de se marier de disposer des cabris dont il a besoin pour constituer la compensation matrimoniale nécessaire à tout mariage. En élevant des cabris les Gbaya disposent de plus, de façon occasionnelle, d'une nourriture conséquente. C'est également un substitut rituel ponctuel de la poule auquel on a recours lorsqu'on veut disposer d'une nourriture consommable par un plus grand nombre de personnes. Il n'y a jamais, en dehors de la situation originelle évoquée dans les contes, de thésaurisation de cabris ni de difficultés à s'en procurer. C'est un bien auquel chacun a accès et qui circule librement entre les familles au gré des naissances et des mariages. Depuis la disparition des barres de fer et de leur remplacement par de l'argent, la chèvre reste le seul élément de la compensation matrimoniale qui manifeste le soutien de la génération des parents vis-à-vis de leurs enfants $^{28}$. Elle est une bonne illustration de la circulation des richesses qui ne dépendent pas des ancêtres.

\section{Références bibliographiques}

Blanchard, Y. et P. Noss, 1982, Dictionnaire gbaya-français, dialecte yaayuwee, Meiganga, Centre de traduction gbaya.

Dorst, J. et P. DANDELot, 1972, Guide des grands mammifères d'Afrique, Neuchatel, Delachaux et Niestlé, $286 \mathrm{p}$.

Letouzet, R., 1972, Manuel de botanique forestière, Afrique tropicale, Paris, Centre technique forestier tropical, 3 tomes, 461 p. : 250, 254

\footnotetext{
${ }^{28}$ Un autre élément très important pour le mariage est le travail du gendre qui, lui, relevait toujours d'un travail individuel du gendre. Or de nos jours, par crainte de voir leur fils sans soutien de la part du lignage (les barres de fer ayant été remplacées par de l'argent) il arrive qu'un père de famille fasse lui-même le travail du gendre au lieu de son fils encore jeune, afin de s'assurer qu'il aura bien les moyens de se marier, même si son père venait à disparaître.
} 
MONINO, Y., 1995 Le proto-gbaya, essai de linguistique comparative sur vingtet-une langues d'Africque centrale, Paris, Peeters, SELAF 357, 725 p.

Roulon Paulette, 1977, Wanto... et l'origine des choses, Contes d'origine et autres contes gbaya-kara (Centrafrique), Paris, Edicef, Coll. Fleuve et flamme, $143 \mathrm{p}$.

Roulon-Doko, P. , 2008, Dictonnaire gbaya-français, suivi d'un dictionnaire des noms propres et d'un index français-gbaya (République Centrafricaine), Paris, Karthala, 696 p.

Roulon-Doko, P., Statut et rôle symbolique des animaux domestiques chez des chasseurs-cueilleurs-cultivateurs: le cas des Gbaya de République Centrafricaine, Réf du CDrom, pp. 553-570.

Roulon-Doko, P., 1...., Parlons gbaya, Paris, l'Harmattan, p.

Roulon-Doko, P., 1998, Chasse, cueillette et cultures chez les Gbaya de Centrafrique, L'Harmattan, Paris, 540 p., 189 figures, 39 photos et 10 cartes.

Roulon-DokO, P., 2001, Cuisine et nourriture chez les gbaya de Centrafricaine, Paris L'Harmattan, $410 \mathrm{p}$.

RoulON-DoKo, P., 2005, La chasse des femmes.... 